[9] —, Supermodularity and Complementarity. Princeton, NJ: Princeton Univ. Press, 1998.

[10] C. Wu and D. Bertsekas, "Distributed power control algorithms for wireless networks," IEEE Trans. Veh. Technol., vol. 50, pp. 504-514, Mar. 2001.

[11] R. D. Yates, "A framework for uplink power control in cellular radio systems," IEEE J. Select. Areas Commun., vol. 13, pp. 1341-1347, Sept. 1995.

[12] D. Yao, "S-modular games with queueing applications," Queueing Syst., vol. 21, pp. 449-475, 1995

\section{Local Stabilization of Linear Systems Under Amplitude and Rate Saturating Actuators}

\author{
João Manoel Gomes da Silva, Jr., Sophie Tarbouriech, and \\ Germain Garcia
}

\begin{abstract}
This note addresses the problem of local stabilization of linear systems subject to control amplitude and rate saturation. Considering the actuator represented by a first-order system subject to input and state saturation, a condition for the stabilization of an a priori given set of admissible initial states is formulated from certain saturation nonlinearities representation and quadratic stability results. From this condition, an algorithm based on the iterative solution of linear matrix inequalities-based problems is proposed in order to compute the control law.
\end{abstract}

Index Terms-Constrained control, control saturation, linear matrix inequality (LMI), stabilization.

\section{INTRODUCTION}

Physical and technological constraints do not allow that control actuators provide unlimited amplitude signals neither react unlimited fast. The negligence of both amplitude and rate control bounds can be source of limit cycles, parasitic equilibrium points and even instability of the closed-loop system. In particular, the problem of stabilization of linear systems only with amplitude saturation has been exhaustively addressed in the literature (see, among others, [1]-[3] and the references therein). On the other hand, the rate saturation problem has first received a special interest in the aeronautic field, where the tradeoff between high performance requirements and the use of hydraulic servos presenting rate limitations is always present (see, for instance, [4], [5], and the references therein).

Studies addressing the stabilization in the presence of both the amplitude and the rate saturation, as a more generic problem, have started to appear in the last few years. In [6] and [7], the semiglobal stabilization of linear systems with both amplitude and rate constraints is addressed. Considering a low-gain approach (the actuator does not effectively saturate), in [6], solutions to the problem via both state feed-

Manuscript received February 16, 2001; revised November 12, 2001 and June 28, 2002. Recommended by Associate Editor T. Iwasaki. This work was supported by an international agreement between CNRS (France) and $\mathrm{CNPq}$ (Brazil).

J. M. Gomes da Silva, Jr. is with the Department of Electrical Engineering, UFRGS, 90035-190 Porto Alegre-RS, Brazil (e-mail: jmgomes@eletro.ufrgs.br).

S. Tarbouriech and G. Garcia are with the L.A.A.S.-C.N.R.S., 31077 Toulouse, cedex 4, France, and also with INSA-Toulouse (e-mail: tarbour@laas.fr; garcia@laas.fr).

Digital Object Identifier 10.1109/TAC.2003.811265 back and observer based output feedback are stated. In [7], the notion of an operator for modeling the amplitude and the rate saturation is introduced. Based on this modeling, a low and high gain approach is used for addressing the problem of semiglobal output regulation via both state and dynamic output feedback. In [8], the problem of external $L_{p}$-stabilization with internal global stabilization via a scheduled low gain (saturation is avoided) state feedback is addressed. It should be pointed out that, since the objective is the semiglobal or global stabilization, these results can be applied only when the open-loop system is null-controllable (i.e., all the poles are in the closed left half plane).

On the other hand, we can identify some works dealing with local stabilizing solutions (see, among others, [9]-[12]). In [9], a method for designing dynamic output controllers based on a position type feedback modeling of the rate saturation and the use of the positive real lemma is proposed. The main objective pursued in that paper is the minimization of a linear quadratic Gaussian criterion. A region of stability (region of attraction) is associated to the closed-loop system. However, it should be pointed out that the size and the shape of this region are not taken into account in the design procedure which can lead to very conservative domains of stability. Furthermore, the controller is computed from the solution of strong coupled equations which, in general, are not simple to solve. A different modeling for the actuator, subject to both rate and amplitude limitations, is considered in [10] and [12]. In these papers, the actuator is modeled by a pure integrator: the control rate appears as the system input and the original control signal becomes a state of the system. The physical meaning of this kind of modeling is not clarified in these papers. Parallel to these works, in [11], the problem of disturbance attenuation in the presence of rate and amplitude actuator saturation is addressed. In that paper, however, no explicitely consideration is made about the region of attraction associated to the controller.

Since we also aim to consider strictly unstable systems, our note figures in the context of local stabilization of linear systems subject to both actuator amplitude and rate saturation. In this case, two objectives are quite natural: the control law should guarantee a certain time-domain performance for the closed-loop system and the associated region of attraction should be as large as possible. Regarding these objectives, a fundamental issue is whether the use of effective saturating control laws can be advantageous or not. In a recent work considering only amplitude saturation [13], it was shown that, at least in some cases, the use of the saturating control laws does not help in obtaining larger regions of stability. It is, however, very important to highlight that no constraints concerning neither the performance, nor the robustness, were taken into account in this analysis. In this case, although the optimal region of stability is obtained with a linear control law, the closed-loop poles associated to this solution can be very close to the imaginary axis, which implies a very slow behavior.

The objective of this note is then to propose a method for computing state feedback saturating control laws, that ensure both asymptotic stability of the closed-loop system with respect to a given set of admissible initial conditions, and a certain degree of time-domain performance in a neighborhood of the origin. We also aim to emphasize the compromise between performance and the size of the region of attraction. As we will see, over performance constraints, the use of saturating control laws can ensure larger regions of stability. As in [9], our approach is based on the modeling of the actuator by a first-order system subject to input and state saturation (position-feedback-type model with speed limitation). Differently, however, from [9] and [11], the objective in the synthesis is explicitely to enlarge the region of attraction. Moreover, the stabilization conditions are based on a mixed polytopic/norm-bounded differential inclusion for modeling the behavior of the closed-loop nonlinear system. Comparing to the polytopic approach used in [11] and 
[12], our approach allows to reduce, considerably, the number of vertices tests. It should be pointed out that, unlike [6], [8], and [10], in our approach effective saturation is allowed and no open-loop stability assumptions are made.

Notations: For any vector $x \in \Re^{n}, x \succeq 0$ means that all the components of $x$, denoted $x_{(i)}$, are nonnegative. For two vectors $x, y$ of $\Re^{n}$, the notation $x \succeq y$ means that $x_{(i)}-y_{(i)} \geq 0, \forall i=1, \ldots, n$. The elements of a matrix $A \in \Re^{m * n}$ are denoted by $a_{(i, l)}, i=1, \ldots, m$, $l=1, \ldots, n . A_{(i)}$ denotes the $i$ th row of matrix $A$. For two symmetric matrices, $A$ and $B, A>B$ means that $A-B$ is positive definite. $A^{T}$ denotes the transpose of $A$. For $x \in \Re^{m}, \mathbb{D}(x)=\operatorname{diag}(x) \in \Re^{m * m}$ denotes a diagonal matrix obtained from vector $x$, i.e., $d_{(i, i)}(x)=x_{(i)}$. $\operatorname{Co}\{\cdot\}$ denotes a convex hull. $1_{m}$ denotes a $m$-dimensional vector of ones, i.e., $1_{m} \triangleq[11, \ldots, 1]^{T} \in \Re^{m}$.

\section{Problem Statement}

Consider the following linear continuous-time system:

$$
\dot{x}(t)=A x(t)+B u(t)
$$

where $x(t) \in \Re^{n}$ and $u(t) \in \Re^{m}$ are, respectively, the state vector and the control vector. Matrices $A$ and $B$ are real constant matrices of appropriate dimensions. The pair $(A, B)$ is supposed to be controllable. Each control actuator is supposed to be a first-order system, that presents both position and rate limitations, that is, $\forall i=1, \ldots, m$ we have

$$
\dot{u}_{(i)}(t)=\operatorname{sat}_{r(i)}\left(\tau_{(i)}\left(-u_{(i)}(t)+\operatorname{sat}_{p(i)}\left(v_{(i)}(t)\right)\right)\right)
$$

where $v_{(i)}$ and $u_{(i)}$ are, respectively, the input and the output (state) of the actuator, $-\tau_{(i)}<0$, corresponds to the pole of the actuator, $\operatorname{sat}_{r(i)}(.) \triangleq \operatorname{sign}(.) \min \left(\xi_{(i)},||.\right), \quad \operatorname{sat}_{p(i)}(.) \triangleq \operatorname{sign}(.) \min \left(\rho_{(i)},||.\right)$ with $\xi_{(i)}$ and $\rho_{(i)}$ denoting, respectively, the rate and amplitude bounds.

Consider now a state feedback defined as follows:

$$
v(t)=K_{x} x(t)+K_{u} u(t) .
$$

Let $T \in \Re^{m * m}$ be a diagonal matrix where $t_{(i, i)}=\tau_{(i)}, i=$ $1, \ldots, m$. The closed-loop system can be described by

$$
\begin{aligned}
& \dot{x}(t)=A x(t)+B u(t) \\
& \dot{u}(t)=\operatorname{sat}_{r}\left(-T u(t)+T \operatorname{sat}_{p}\left(K_{x} x(t)+K_{u} u(t)\right)\right) .
\end{aligned}
$$

Define the regions $R_{L_{p}}$ and $R_{L r}$, respectively, as the region where there is no occurrence of amplitude saturation and the region where there is no occurrence of rate saturation

$$
\begin{aligned}
& R_{L p} \triangleq\left\{x \in \Re^{n}, u \in \Re^{m} ;\left|\left[\begin{array}{ll}
K_{x} & K_{u}
\end{array}\right]\left[\begin{array}{l}
x \\
u
\end{array}\right]\right| \preceq \rho\right\} \\
& R_{L r} \triangleq\left\{x \in \Re^{n}, u \in \Re^{m} ;\left|-T u+T \operatorname{sat}_{p}\left(K_{x} x+K_{u} u\right)\right| \preceq \xi\right\} .
\end{aligned}
$$

It follows that $R_{L} \triangleq R_{L p} \cap R_{L r}$ is the region of linear behavior of (1), i.e., where no saturation occurs.

Define now the augmented state vector $z(t) \triangleq\left[\begin{array}{l}x(t) \\ u(t)\end{array}\right] \in \Re^{n+m}$. Let $\mathcal{Z}_{0}$ be a set of admissible initial states in the state space $\Re^{n+m}$. This set can be viewed as the zone of operation of (4). From the aforementioned definitions and considerations, the problem we intend to solve is stated as follows.

Problem 1: Find matrices $K_{x}$ and $K_{u}$ such that the following hold.

1) System (4) is locally asymptotically stable in $\mathcal{Z}_{0}$, that is, $\forall z(0)=\left[x(0)^{T} u(0)^{T}\right]^{T} \in \mathcal{Z}_{0}$, the corresponding trajectories converge asymptotically to the origin.

2) When the system operates inside the linearity region $R_{L}$, a certain time-domain performance specification is satisfied.
Remark 1: The model of rate-limiting we consider, is known as the classical rate limiter and can be viewed as a position-feedback-type model with speed limitation [9]. As pointed in [9], for $\tau_{(i)} \rightarrow \infty$, this model corresponds to the ideal rate limiter of Simulink. This case corresponds also to the rate operator defined in [7].

\section{SYSTEM REPRESENTATION}

In order to carry out a solution to Problem 1, in this section we deduce a locally valid representation for system (4). The first step consists in rewriting the saturation terms as varying parameters that depend on the state of the system at each instant. For this, consider

- a vector $\alpha(t) \in \Re^{m}$ with $\alpha_{(i)}(t) \triangleq \min \left(1, \rho_{(i)} / \mid K_{x(i)} x(t)+\right.$ $\left.K_{u(i)} u(t) \mid\right) i=1, \ldots, m$;

- a vector $\beta(t) \in \Re^{m}$ with $\beta_{(i)}(t) \triangleq \min \left(1, \xi_{(i)} / \mid-T_{(i)} u(t)+\right.$ $\left.T_{(i)} \operatorname{sat}_{p} K_{x} x(t)+K_{u} u(t) \mid\right) i=1, \ldots, m$.

From these definitions, it follows that $\operatorname{sat}_{p}(v(t))=\mathbb{D}(\alpha(t))\left(K_{x} x(t)+K_{u} u(t)\right)$ and $\operatorname{sat}_{r}\left(-T u(t)+\operatorname{Tsat}_{p}\left(K_{x} x(t)+K_{u} u(t)\right)=\right.$ $\mathbb{D}(\beta(t))\left(-T u(t)+T \mathbb{D}(\alpha(t))\left(K_{x} x(t)+K_{u} u(t)\right)\right)$. Hence, the closed-loop system (4) is equivalent to the following one:

$$
\dot{z}(t)=(\mathcal{A}(\beta(t))+\mathcal{B}(\beta(t)) \mathbb{D}(\alpha(t)) \mathcal{K}) z(t)
$$

where

$$
\begin{aligned}
\mathcal{A}(\beta(t)) & =\left[\begin{array}{cc}
A & B \\
0 & -\mathbb{D}(\beta(t)) T
\end{array}\right] \\
\mathcal{B}(\beta(t)) & =\left[\begin{array}{c}
0 \\
\mathbb{D}(\beta(t)) T
\end{array}\right] \\
\mathcal{K} & =\left[\begin{array}{ll}
K_{x} & K_{u}
\end{array}\right] .
\end{aligned}
$$

In particular, if $z(t)$ belongs to the linearity region $R_{L}$ it follows that $\alpha(t)=\beta(t)=1_{m}$ and the behavior of the system is given by the linear equation

$$
\dot{z}(t)=(\mathcal{A}+\mathcal{B K}) z(t)
$$

with $\mathcal{A}=\left[\begin{array}{cc}A & B \\ 0 & -T\end{array}\right]$ and $\mathcal{B}=\left[\begin{array}{l}0 \\ T\end{array}\right]$.

Consider now that $z(t)$ belongs to some region in the state space, not contained in $R_{L}$, such that

$$
\begin{aligned}
& 0<\underline{\alpha}_{(i)} \leq \alpha_{(i)}(t) \leq 1 \\
& 0<\underline{\beta}_{(i)} \leq \beta_{(i)}(t) \leq 1
\end{aligned} \quad \forall i=1, \ldots, m
$$

where $\underline{\alpha}_{(i)}$ and $\underline{\beta}_{(i)}$ are lower bounds for $\alpha_{(i)}(t)$ and $\beta_{(i)}(t)$. From convexity arguments, for all $z(t)$ belonging to the considered region it follows that: $\mathbb{D}(\alpha(t)) \in \mathbf{C o}\left\{\mathbb{D}_{1}(\underline{\alpha}), \mathbb{D}_{2}(\underline{\alpha}), \ldots, \mathbb{D}_{2} m(\underline{\alpha})\right\}$ and $\mathbb{D}(\beta(t)) \in \mathbf{C o}\left\{\mathbb{D}_{1}(\beta), \mathbb{D}_{2}(\beta), \ldots, \mathbb{D}_{2} m(\beta)\right\}$ where $\mathbb{D}_{j}(\underline{\alpha})$ (respectively $\mathbb{D}_{j}(\beta)$ ) are the vertices of a polytope of diagonal matrices whose diagonal elements can assume the value 1 or $\underline{\alpha}_{(i)}$ (respectively, $\left.\underline{\beta}_{(i)}\right), i=1, \ldots, m$. From the matrices $\mathbb{D}_{j}(\underline{\beta}), j=1, \ldots, 2^{m}$, define $\mathcal{A}_{j}(\underline{\beta})=\left[\begin{array}{cc}A & B \\ 0 & -\mathbb{D}_{j}(\beta) T\end{array}\right]$ and $\mathcal{B}_{j}(\underline{\beta})=\left[\begin{array}{c}0 \\ \mathbb{D}_{j}(\beta) T\end{array}\right]$.

Now, from the previous definitions, we state $\bar{a}$ lemma that, as we will see in the next section, will allow to use a mixed polytopic/normbounded differential inclusion in order to conclude about the local stability of (4).

Lemma 1: Consider a vector $\underline{\alpha} \in \Re^{m}$ and a vector $\underline{\beta} \in \Re^{m}$ whose components $\underline{\alpha}_{(i)}, \underline{\beta}_{(i)}, i=1, \ldots, m$ belong to the interval $(0,1]$, and define the following polyhedral sets:

$$
\begin{aligned}
& R_{L p}(\underline{\alpha})=\left\{z \in \Re^{n+m} \quad|\mathcal{K} z| \preceq \rho(\underline{\alpha})\right\} \\
& R_{L r}(\underline{\beta})=\cap_{j=1}^{2^{m}} R_{L r}(\underline{\beta})_{j}
\end{aligned}
$$


where $\quad R_{L r}(\beta)_{j} \triangleq\left\{z \quad \in \quad \Re^{n+m} ; \quad \mid\left[T \mathbb{D}_{j}(\underline{\alpha}) K_{x} \quad(-T \quad+\right.\right.$ $\left.\left.\left.T \mathbb{D}_{j}(\underline{\alpha}) K_{u}\right)\right] z \mid \quad \preceq \quad \xi(\underline{\beta})\right\} \quad$ with $\rho_{(i)}(\underline{\alpha}) \triangleq\left(\rho_{(i)} / \underline{\alpha}_{(i)}\right)$, $\xi_{(i)}(\beta) \triangleq\left(\xi_{(i)} / \underline{\beta}_{(i)}\right), \forall i=1, \ldots, m$.

If $z(t) \in R_{L p}(\underline{\alpha}) \cap R_{L r}(\underline{\beta})$, then $\dot{z}(t)$ can be computed as

$$
\sum_{j=1}^{2^{m}} \lambda_{j}(z(t))\left[\left(\mathcal{A}_{j}(\underline{\beta})+\mathcal{B}_{j}(\underline{\beta}) \Gamma_{1}(\underline{\alpha}) \mathcal{K}\right)+\mathcal{B}_{j}(\underline{\beta}) \mathbb{D}(\theta(t)) \mathcal{K}\right] z(t)
$$

with $\Gamma_{1}(\underline{\alpha}) \triangleq \mathbb{D}\left(0.5\left(1_{m}+\underline{\alpha}\right)\right),\left|\theta_{(i)}(t)\right| \leq 0.5\left(1-\underline{\alpha}_{(i)}\right), \lambda_{j}(z(t)) \geq 0$, $\forall j=1, \ldots, 2^{m}$ and $\sum_{j=1}^{2^{m}} \lambda_{j}(z(t))=1$.

Proof: For all $z(t) \in R_{L p}(\underline{\alpha}) \cap R_{L r}(\beta)$, one satisfies (7). Consider now an auxiliary vector $\theta(\bar{t})$ such that $\theta_{(i)}(t)=\alpha_{(i)}(t)-1+0.5\left(1-\underline{\alpha}_{(i)}\right) i=1, \ldots, m$. Hence, $\forall z(t) \in R_{L p}(\underline{\alpha})$, it follows that $\left|\theta_{(i)}(t)\right| \leq 0.5\left(1-\underline{\alpha}_{(i)}\right)$ and we can conclude that $\dot{z}(t)$ can be computed by $\dot{z}(t)=$ $\left(\mathcal{A}(\beta(t))+\mathcal{B}(\beta(t)) \Gamma_{1}(\underline{\alpha}) \mathcal{K}\right) z(t)+\mathcal{B}(\beta(t)) \mathbb{D}(\theta(t)) \mathcal{K} z(t)$ with $\left|\theta_{(i)}(t)\right| \leq 0.5\left(1-\underline{\alpha}_{(i)}\right), \Gamma_{1}(\underline{\alpha}) \triangleq \mathbb{D}\left(0.5\left(1_{m}+\underline{\alpha}\right)\right)=$ $\mathbb{D}(\alpha(t))-\mathbb{D}(\theta(t))$.

Considering the matrices $\mathbb{D}_{j}(\beta)$, defined from the vector $\underline{\beta}$, it follows that the matrices $\mathcal{A}_{j}(\beta)$ and $\mathcal{B}_{j}(\beta)$ are the vertices of convex polytopes of matrices. It follows that at instant $t$, if $z(t) \in R_{L p}(\underline{\alpha}) \cap R_{L r}(\beta)$ there exist scalars $\lambda_{j}(z(t)) \geq 0$, $\forall j=1, \ldots, 2^{m}, \sum_{j=1}^{2^{m}} \lambda_{j}(\bar{z}(t))=1$, such that $\dot{z}(t)$ can be computed

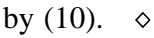

\section{MAIN RESUltS}

In order to solve Problem 1, we should compute a state feedback that guarantees the local stability of system (4) in a region that contains the set $\mathcal{Z}_{0}$. Furthermore, when this system operates in the region of linearity, i.e., the closed-loop system is described by (6), a certain degree of time-domain performance should be guaranteed. This kind of specification can, in general, be achieved by placing the poles of $(\mathcal{A}+\mathcal{B K})$ in a suitable region of the half left complex plane. Hence, consider the following data:

- a set of initial conditions $\mathcal{Z}_{0}$ defined by a polyhedral set in $\Re^{n+m}$ described by its vertices

$$
\mathcal{Z}_{0} \triangleq \mathbf{C o}\left\{v_{1}, \ldots, v_{n_{v}}\right\} \quad v_{s} \in \Re^{n+m} \quad \forall s=1, \ldots, n_{n_{v}}
$$

- a region $\mathcal{D}_{p}$, contained in the left-half complex plane

$$
\mathcal{D}_{p} \triangleq\left\{s \in \mathcal{C} ; \quad\left(H+s G+\bar{s} G^{T}\right)<0\right\}
$$

where $H=H^{T} \in \Re^{l * l}, G \in \Re^{l * l}$ and $s$ is a complex number with its conjugate $\bar{s}$. This is an LMI region as defined in [14]. We assume that if the poles of $(\mathcal{A}+\mathcal{B K})$ are located in the region $\mathcal{D}_{p}$, the time-domain performance specifications in the linearity region of (4) are satisfied.

Hence, considering this data, if we are able to find a matrix $\mathcal{K}$, vectors $\underline{\alpha}, \beta$, and a set $\mathcal{E}$ in the state-space such that the set $\mathcal{E}$ is contractive with respect to the trajectories of the differential inclusion (10), and $\mathcal{Z}_{0} \subset \mathcal{E} \subset\left(R_{L p}(\underline{\alpha}) \cap R_{L r}(\beta)\right)$, then we can conclude that all the trajectories of the saturated system (4) starting in $\mathcal{E}$ (and, in consequence, all the trajectories starting in $\mathcal{Z}_{0}$ ) converge asymptotically to the origin. In this case, the set $\mathcal{E}$ is a domain of asymptotic stability for (4). If, in addition, the poles of $(\mathcal{A}+\mathcal{B K})$ are contained in $\mathcal{D}_{p}$, Problem 1 is solved. These ideas are formalized in the following proposition. For notational simplicity we consider: $\mathcal{A}_{j}(\beta)=\mathcal{A}_{j}, \mathcal{B}_{j}(\beta)=\mathcal{B}_{j}$ and $\lambda_{j}(z(t))=\lambda_{j}$.
Proposition 1: If there exist a matrix $W=W^{T}>0, W \in$ $\Re^{(n+m) *(n+m)}$, a diagonal matrix $S>0, S \in \Re^{m * m}$, a matrix $Y \in \Re^{m *(n+m)}$, and vectors $\underline{\alpha} \in \Re^{m}$ and $\beta \in \Re^{m}$, satisfying the following matrix inequalities:
i) $\left[\begin{array}{cc}M_{j} & \star \\ \Gamma_{2}(\underline{\alpha}) Y & S\end{array}\right]>0, j=1, \ldots, 2^{m}$;
ii) $\left[\begin{array}{cc}W & \star \\ \underline{\alpha}_{(i)} Y_{(i)} & \rho_{(i)}^{2}\end{array}\right] \geq 0, i=1, \ldots, m$;
iii) $\left[\begin{array}{cc}1 & \star \\ v_{s} & W\end{array}\right] \geq 0, s=1, \ldots, n_{v}$;

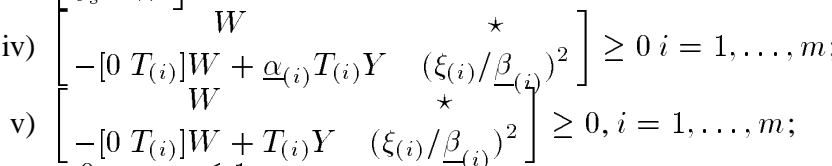
vi) $\left\{\begin{array}{l}0<\underline{\alpha}_{(i)} \leq 1 \\ 0<\underline{\beta}_{(i)} \leq 1\end{array} i=1, \ldots, m ;\right.$
vii) $h_{(i, j)} W+g_{(i, j)}(\mathcal{A} W+\mathcal{B} Y)+g_{(i, j)}(\mathcal{A} W+\mathcal{B} Y)^{T}<0$ $1 \leq i, j \leq l$

where $M_{j}=-W \mathcal{A}_{j}^{T}-\mathcal{B}_{j} \Gamma_{1}(\underline{\alpha}) Y-\mathcal{A}_{j} W-Y^{T} \Gamma_{1}(\underline{\alpha}) \mathcal{B}_{j}^{T}-\mathcal{B}_{j} \mathcal{S} \mathcal{B}_{j}^{T}$, $\Gamma_{1}(\underline{\alpha}) \triangleq \mathbb{D}\left(0.5\left(1_{m}+\underline{\alpha}\right)\right), \Gamma_{2}(\underline{\alpha}) \triangleq \mathbb{D}\left(0.5\left(1_{m}-\underline{\alpha}\right)\right)$, then $\mathcal{K} \triangleq Y W^{-1}$ solves Problem 1.

Proof: Consider the set $\mathcal{E} \triangleq\left\{z \in \Re^{n+m} ; z^{T} P z \leq 1\right\}$ with $P \triangleq W^{-1}$ and the regions $R_{L p}(\underline{\alpha})$ and $R_{L r}(\beta)$ defined, respectively, in (8) and (9). Consider also $\mathcal{K}=Y W^{-1}$.

Satisfaction of conditions i)-vii) leads to the following facts.

1) Pre- and postmultiplying i) by $\left[\begin{array}{ll}P & 0 \\ 0 & I\end{array}\right]$, considering $\mathcal{K}=Y W^{-1}$ and applying Schur's complement it follows that i) is equivalent to $\left[\begin{array}{cc}L_{j}-\mathcal{K}^{T} \Gamma_{2}(\underline{\alpha}) S^{-1} \Gamma_{2}(\underline{\alpha}) \mathcal{K} & P \mathcal{B}_{j} \\ B_{j}^{T} P & S^{-1}\end{array}\right]>0$, where $L_{j}=-\left(\mathcal{A}_{j}+\mathcal{B}_{j} \Gamma_{1}(\underline{\alpha}) \mathcal{K}\right)^{T} P-P\left(\mathcal{A}_{j}+\right.$ $\left.\mathcal{B}_{j} \Gamma_{1}(\underline{\alpha}) \mathcal{K}\right)$. Since i) holds for $j=1, \ldots, 2^{m}$, by convexity one obtains $\sum_{j=1}^{2^{m}} \lambda_{j}\left(z^{T} L_{j} z+z^{T} P \mathcal{B}_{j} q+\right.$ $\left.q^{T} \mathcal{B}_{j}^{T} P z-\sum_{i=1}^{m} \sigma_{(i)}\left[z^{T} \underline{\theta}_{(i)} \mathcal{K}_{(i)}^{T} \mathcal{K}_{(i)} \underline{\theta}_{(i)} z-q_{(i)}^{2}\right]\right)>0$, $\forall z, q$ and $\lambda_{j} \geq 0, j=1, \ldots, m$, such that $\sum_{j=1}^{2^{m}} \lambda_{j}=1$, with $\sigma_{(i)}$ denoting the $i^{\text {th }}$ diagonal component of $S^{-1}$ and $\underline{\theta}_{(i)}=0.5\left(1-\underline{\alpha}_{(i)}\right)$.

2) ii) and iv)-vi) ensure that $\mathcal{E} \subset R_{L p}(\underline{\alpha}) \cap R_{L r}(\beta)$ [15], [16].

3) iii) ensures that $\mathcal{Z}_{0} \subset \mathcal{E}[16]$

4) vii) ensures that the poles of $(\mathcal{A}+\mathcal{B K})$ are placed in the region $\mathcal{D}_{p}$ defined in (12) [14].

From Lemma 1 , if $z(t) \in\left(R_{L p}(\underline{\alpha}) \cap R_{L r}(\beta)\right)$, then there exist $\lambda_{j} \geq 0, j=1, \ldots, 2^{m}$, with $\sum_{j=1}^{2^{m}} \lambda_{j}=1$, such that $\dot{z}(t)$ can be computed by (10) with $\left|\theta_{(i)}(t)\right| \leq \underline{\theta}_{(i)}$. Suppose now that $z(t) \in \mathcal{Z}_{0}$. Since ii)-v) are verified, we have that $z(t) \in \mathcal{E} \subset\left(R_{L p}(\underline{\alpha}) \cap R_{L r}(\beta)\right)$, and $\left|\theta_{(i)}(t)\right| \leq \underline{\theta}_{(i)}, i=1, \ldots, m$. Therefore, $\dot{z}(t)$ can be computed by (10). Consider now that $q(t)=-\mathbb{D}(\theta(t)) \mathcal{K} z(t)$. Since $\left|\theta_{(i)}(t)\right| \leq \underline{\theta}_{(i)}$, it follows that $z(t)^{T} \underline{\theta}_{(i)} \mathcal{K}_{(i)}^{T} \mathcal{K}_{(i)} \underline{\theta}_{(i)} z(t) \geq q_{(i)}^{2}(t)$. Hence, from fact 1) and taking into account that $\sigma_{(i)}>0$, one obtains $\sum_{j=1}^{2^{m}} \lambda_{j} z(t)^{T} L_{j} z(t)-$ $z(t)^{T} P \sum_{j=1}^{2^{m}} \lambda_{j} \mathcal{B}_{j} \mathbb{D}(\theta(t)) \mathcal{K} z(t)-z(t)^{T} \mathcal{K}^{T} \mathbb{D}(\theta(t)) \sum_{j=1}^{2^{m}} \lambda_{j} \mathcal{B}_{j}^{T}$ $P z(t)>0$. Therefore, from (10), one has $\dot{z}(t)^{T} P z(t)+$ $z(t)^{T} P \dot{z}(t)<0$.

Since this reasoning is valid $\forall z(t) \in \mathcal{E}$, we can conclude that $\mathcal{E}$ is a positively invariant and contractive set w.r.t (5) [or, equivalently, (4)] and $V(z(t))=z^{T}(t) P z(t)$ is a strictly decreasing Lyapunov function for (5) [or, equivalently, (4)]. Hence, we can conclude that if conditions i)-vi) are satisfied with $\mathcal{K}=Y W^{-1}$, it follows that $\forall z(0) \in \mathcal{Z}_{0}$ the corresponding trajectory does not leave $\mathcal{E}$ and converges asymptotically to the origin. Furthermore, the poles of $(\mathcal{A}+\mathcal{B K})$ are placed in 
$\mathcal{D}_{p}$ guaranteeing the performance specification in the linearity region $R_{L}$. Problem 1 is then solved if conditions i)-vii) are fulfilled. $\diamond$

Remark 2: Proposition 2 gives only a sufficient condition for the solution of Problem 1. The main sources of conservatism here are the use of quadratic Lyapunov functions and the modeling of the system by a differential inclusion. Note that all the trajectories of (4) in $\mathcal{E}$ are trajectories of (10) but the converse is not true.

Remark 3: Although our main concern is to ensure stability when the actuators saturate, a certain degree of time-domain performance can be also considered when the system operates in the nonlinear region. For example, it can be considered an eigenvalue shift in i) in order to ensure a convergence rate and improve robustness. However, it should be pointed out that it is not realistic to impose the same performance requirements for the system over the linear and the saturated actuator region.

\section{COMPutation OF THE CONTROL LaW}

The variables to be found by applying Proposition 1 are $W, Y, S$, $\underline{\alpha}$ and $\beta$. Due to terms involving products between these variables, inequalities i), ii), iv), and v) of Proposition 1 are nonlinear, whereas relations iii), vi), and vii) are linear (i.e., they are LMIs). This fact implies that the attempt to compute $\mathcal{K}$ by solving constraints i)-vii) directly as a feasibility problem, in the variables $W, Y, S, \underline{\alpha}$, and $\beta$, is very difficult (it is an NP-hard problem) or even impossible.

A way to overcome this problem is to fix, a priori, the value of the components of $\underline{\alpha}$ [11], [15] and $\beta$. In this case, all inequalities become LMIs and, given $\left(\mathcal{Z}_{0}, \mathcal{D}_{p}\right)$, it is possible to solve constraints i)-vii) of Proposition 1, as a feasibility problem, with efficient numerical algorithms [16]. Of course, considering the fixed vectors $\underline{\alpha}$ and $\beta$ and the given data, it may actually be impossible to find a feasible solution. In fact, considering a scaling factor $\delta, \delta>0$, the maximum homothetic set to $\mathcal{Z}_{0}$, denoted $\delta^{\star} \mathcal{Z}_{0}$, that can be stabilized using the proposed approach, considering the fixed $\underline{\alpha}$ and $\beta$, can be obtained by solving the following convex optimization problem with LMI constraints:

$$
\begin{gathered}
\max \delta \\
\left\{\begin{array}{cc}
{\left[\begin{array}{cc}
1 & \delta v_{s}^{T} \\
\delta v_{s} & W
\end{array}\right]>0 \quad \forall s=1, \ldots, n_{v}} \\
\text { i),ii),iv),v), and vii) of Proposition } 1 .
\end{array}\right.
\end{gathered}
$$

Hence, if the optimal value of $\delta, \delta^{\star}$, is greater or equal to 1 , it means that it is possible to find a solution considering the fixed $\underline{\alpha}$ and $\beta$ for the given data $\left(\mathcal{Z}_{0}, \mathcal{D}_{p}\right)$.

If a linear solution for Problem 1 is desired, that is, if one does not want to excite the saturation nonlinearities, it suffices to consider $\underline{\alpha}=$ $\beta=1_{m}$ in the optimization problem (13). In this case, $\mathcal{A}_{j}=\mathcal{A}$, $\overline{\mathcal{B}}_{j}=\mathcal{B}, \forall j=1, \ldots, 2^{m} ; \Gamma_{1}(\underline{\alpha})=I_{m}, \Gamma_{2}(\underline{\alpha})=0$ The optimal solution for (13) obtained with $\underline{\alpha}=\beta=1_{m}$ corresponds then to the larger $\delta$ achievable with a linear controller, i.e., the larger region $\delta \mathcal{Z}_{0}$, for which it is possible to ensure quadratic stability with a linear control law $v(t)=\mathcal{K} z(t)=Y W^{-1} z(t)$. On the other hand, we conjecture that the choice of the components of $\underline{\alpha}$ and $\beta$ smaller than 1 can be useful in order to obtain a greater value for $\delta$ in (13). In other words, the degree of freedom in the choice of $\underline{\alpha}$ and $\beta$ can be explored to stabilize larger domains of admissible initial states in the presence of the pole placement constraints (see the numerical example). In fact, smaller are the components of $\underline{\alpha}$ and $\beta$, larger is the region $\left(R_{L p}(\underline{\alpha}) \cap R_{L p}(\beta)\right)$ where the invariant ellipsoid containing $\mathcal{Z}_{0}$ can be included.

In this case, two issues arise: how to choose the initial vectors $\underline{\alpha}$ and $\beta$ and how exactly to decrease the components of $\underline{\alpha}$ and $\beta$ (if $\delta^{\star}<1$ ).
One simple way of handling these issues is to apply trial and error procedures. In particular, for the single-input case, it is possible to seek the optimal solution of (13) over a grid on $\underline{\alpha}$ and $\beta$. For the general multiple-input case, we can seek for a suboptimal solution by using an iterative algorithm, where in each step, two or three variables are fixed and a convex optimization problem with LMI constraints is solved.

Algorithm 1:

- Step 1: Fix $\underline{\alpha}$ and $\beta$, solve (13) for $W, Y, S$, and $\delta$.

- Step 2: Fix $\beta$ and $\bar{Y}$, solve (13) for $W, S, \underline{\alpha}$, and $\delta$.

- Step 3: Fix $\bar{W}, Y, S$, and $\underline{\alpha}$ solve the following optimization problem:

$$
\min _{\underline{\underline{\beta}}} \sum_{i=1}^{m} \underline{\beta}_{\text {subject to }}
$$

i),iv),v), and vi) of Proposition 1.

The iteration between these three steps stops when a desired precision for $\delta$ is achieved. If $\delta^{\star} \geq 1$, it means that it is possible to stabilize (4) for all initial conditions in $\mathcal{Z}_{0}$ by considering the pole placement of $(\mathcal{A}+\mathcal{B} \mathcal{K})$ inside $\mathcal{D}_{p}$. In particular, all intermediate solutions with $\delta>1$ are solutions to Problem 1 . In this case, another optimization scheme can be used in order to select a gain, under a performance criterion (see, for example, [3]). Although conservative (in the sense that, in general, the optimal solution is not achieved) this kind of approach solves, in part, the problem of the choice of vectors $\underline{\alpha}$ and $\beta$ by using robust and available packages to solve LMIs [17]. Furthermore, it is worth noticing that since pair $(A, B)$ is controllable, there will always exist a solution for (13) for $\underline{\alpha}=\beta=1_{m}$. Hence, if we start the algorithm with $\underline{\alpha}=\beta=1_{m}$, the convergence to a solution $\left(W^{\star}, Y^{\star}\right.$, $\left.S^{\star}, \underline{\alpha}^{\star}, \beta^{\star}, \delta^{\star}\right)$ is ensured. This follows from the fact that an optimal solution for one step, is also a feasible solution for the next step. Of course, taking different initial vectors $\underline{\alpha}$ and $\beta$, the proposed algorithm can converge to different values of $\left(\bar{W}^{\star}, \overline{Y^{\star}}, S^{\star}, \underline{\alpha}^{\star}, \beta^{\star}, \delta^{\star}\right)$. Furthermore, other relaxation schemes can be considered. For example, a fourth step considering the minimization of the sum of $\underline{\alpha}_{(i)}$, could be added to the algorithm.

Remark 4: Although we claim that the fact of taking smaller values for $\underline{\alpha}_{(i)}$ and $\underline{\beta}_{(i)}$ can lead to obtain larger $\delta$ in the presence of the pole placement constraint, it does not mean that the components of $\underline{\alpha}$ and $\beta$ can be indefinitely decreased. In fact, considering $\underline{\alpha}$ and $\beta$ as free variables in (13), the optimal $\delta$ is not obtained for $\underline{\alpha}_{(i)} \rightarrow 0$ and $\underline{\beta}_{(i)} \rightarrow$ 0 (see examples in Section VI). Furthermore, note that if $\underline{\alpha}_{(i)}$ and $\underline{\beta}_{(i)}$ are excessively reduced, (13) may be not feasible. This can be justified by the following.

- We consider a differential inclusion for representing the behavior of the nonlinear system. Hence, implicitly, we are somewhat dealing with the stabilization of an uncertain system. In this case, the uncertainty degree is greater for smaller $\underline{\alpha}_{(i)}$ and $\underline{\beta}_{(i)}$.

- Considering strictly unstable open-loop systems it is well known that the global stabilization cannot be achieved. Considering $\underline{\alpha}_{(i)} \rightarrow 0$ and $\underline{\beta}_{(i)} \rightarrow 0, R_{L(\underline{\alpha})} \cap R_{L(\underline{\alpha})}$ would approach the whole state-space, however the region of attraction would be limited.

Remark 5: It should be pointed out that, during the review process of this note, a conference paper addressing the problem of linear systems with nested saturations has been published [18]. It appears that the approach proposed in that paper (similar, in the principle, to our first results concerning the rate saturation problem published in [19]) can provide a way of avoiding the nonlinearities and the relaxation schemes, by a direct LMI formulation. 
TABLE I

$\rho=1, \xi=10$

\begin{tabular}{c|c|c|c|c|c}
\hline$\mu$ & $\delta_{\text {lin }}$ & $\underline{\underline{\alpha}}^{\star}$ & $\beta^{\star}$ & $\delta^{\star}$ & eig $_{\max }$ \\
\hline 0 & 0.044 & 1 & 0.4 & 0.047 & -0.345 \\
\hline 1 & 0.034 & 0.8 & 0.4 & 0.046 & -1.44 \\
\hline 5 & 0.010 & 0.7 & 0.4 & 0.014 & $-7.14 \pm 1.27$ \\
\hline
\end{tabular}

\section{EXAMPLES AND CONCLUDING REMARKS}

Example 1: Let (1) be described by the following matrices:

$$
A=\left[\begin{array}{cc}
0 & 1 \\
10 & -0.1
\end{array}\right] \quad B=\left[\begin{array}{l}
0 \\
1
\end{array}\right] \quad T=20 .
$$

Let the set of admissible initial conditions be given by an hypercube in $\Re^{3}: \mathcal{Z}_{0}=\left\{z \in \Re^{3} ;-1 \leq z_{(i)} \leq 1, \forall i=1, \ldots, 3\right\}$. Given these data, the objective is to solve Problem 1 . We consider that the performance specification in the linearity region was translated as the placement of the poles of $(\mathcal{A}+\mathcal{B K})$ in the region $\mathcal{D}_{p}=\{s \in \mathcal{C} ; \operatorname{Re}\{s\}<$ $-\mu, \mu>0\}$. Notice that the greater is $\mu$, the farther from the origin are the poles of $(\mathcal{A}+\mathcal{B K})$, and greater tends to be the speed of the convergence of the trajectories to the origin inside the region of linearity.

Considering $\rho=1, \xi=10$ and the scaling factor $\delta$, Table I shows the value of $\delta\left(\delta_{\text {lin }}\right)$ obtained in the linear case (i.e. for $\underline{\alpha}=1$ and $\beta=1$ ), and optimal values of $\underline{\alpha}\left(\underline{\alpha}^{\star}\right), \beta\left(\beta^{\star}\right)$ and $\delta\left(\delta^{\star}\right)$, obtained from the solution of (13) considering different values for $\mu$. eig $g_{\max }$ denotes the maximal eigenvalue of $(\mathcal{A}+\mathcal{B} \mathcal{K})$ corresponding to the optimal solution. ${ }^{1}$

The following facts can be noticed.

- If no performance constraint is considered $(\mu=0)$, the best value of $\delta$ is obtained. However, the poles of $(\mathcal{A}+\mathcal{B K})$ are very close to the imaginary axis.

- For $\mu \neq 0$, the best solution is obtained in the saturated case. Note that the optimal values for $\underline{\alpha}$ and $\beta$ are smaller than 1 . For instance, the optimal $\delta$ obtained for $\mu=5$ is about $40 \%$ larger than the one obtained in the saturation avoidance case (i.e., for $\underline{\alpha}=1$ and $\beta=1$ ). This means that the proposed approach allows to obtain solutions to Problem 1 for larger sets of admissible initial states when performance (or robustness) constraints are considered.

Considering the case with $\mu=0.5$, one obtains

$$
W=\left[\begin{array}{ccc}
0.0048 & -0.0238 & 0.0501 \\
-0.0238 & 0.1381 & -0.4387 \\
0.0501 & -0.4387 & 3.1613
\end{array}\right]
$$

Fig. 1 depicts the control response for this case considering two initial conditions belonging to the stability set $\mathcal{E}$. It can be noticed the effective saturation of $\dot{u}(t)$ and $v(t)$.

Consider now the case without rate saturation $(\xi=\infty)$. Table II depicts the optimal values for the optimization problem (13). The maximum $\delta$ is obtained for the linear case $(\underline{\alpha}=1)$ with $\mu=0$. This is in accordance with the result of [13]. Note, however, that in this case the eigenvalues of the linear system are very close to the imaginary axis. Considering $\mu \neq 0$, the best $\delta$ is achieved considering saturation, i.e., $\underline{\alpha} \neq 1$.

${ }^{1}$ Since the system is single-input the optimal solution can be approached with a desired precision by a gridding procedure.

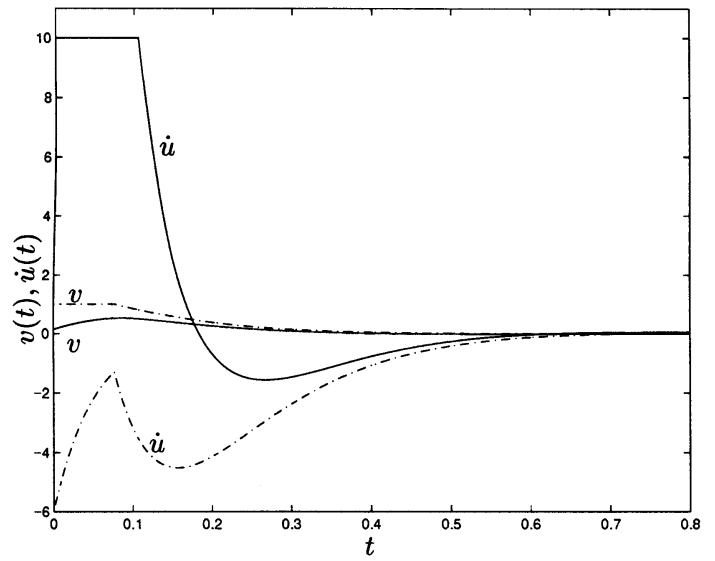

Fig. 1. Simulation results considering: $z(0)=[0.0326-$

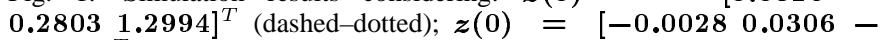
$1.0440]^{T}$ (continuous).

TABLE II

No-RATE CONSTRAINTS, $\boldsymbol{\rho}=1$

\begin{tabular}{c|c|c|c|c}
\hline$\mu$ & $\delta_{l i n}$ & $\underline{\alpha}^{\star}$ & $\delta^{\star}$ & $e i g_{\max }$ \\
\hline 0 & 0.0653 & 1 & 0.0653 & -0.0001 \\
\hline 1 & 0.0494 & 0.76 & 0.0653 & -1.005 \\
\hline 5 & 0.0133 & 0.49 & 0.0266 & $-5.08 \pm j 3.09$ \\
\hline
\end{tabular}

TABLE III

ALgORITHM PERformance

\begin{tabular}{c|c|c|c|c|c}
\hline$\mu$ & $\delta_{\text {lin }}$ & \multicolumn{2}{|c|}{$\underline{\underline{\alpha}}^{T}, \beta^{T}$} & $\delta$ & $e i g_{\max }$ \\
\hline 0 & 10.40 & {$\left[\begin{array}{ll}1 & 1\end{array}\right],\left[\begin{array}{ll}1 & 1\end{array}\right]$} & 10.40 & $-0.001 \pm j 0.47$ \\
\hline 0.3 & 2.93 & {$\left[\begin{array}{lll}1 & 0.952\end{array}\right],\left[\begin{array}{ll}0.847 & 0.208\end{array}\right]$} & 3.60 & $-0.31 \pm j 0.08$ \\
\hline 0.5 & 1.27 & {$\left[\begin{array}{lll}1 & 0.935\end{array}\right],\left[\begin{array}{ll}0.831 & 0.323\end{array}\right]$} & 1.54 & $-0.52 \pm j 0.15$ \\
\hline 1 & 0.34 & {$\left[\begin{array}{ll}1 & 0.954\end{array}\right],\left[\begin{array}{ll}0.763 & 0.425\end{array}\right]$} & 0.397 & $-1.02 \pm j 0.32$ \\
\hline
\end{tabular}

Example 2: Consider the matrices of the system given by [12]

$$
\begin{aligned}
A & =\left[\begin{array}{cccc}
-0.0366 & 0.0271 & 0.0188 & -0.4555 \\
0.0482 & -1.0100 & 0.0024 & -4.0208 \\
0.1002 & 0.2855 & -0.7070 & 1.3230 \\
0 & 0 & 1.0000 & 0
\end{array}\right] \\
B & =\left[\begin{array}{cc}
0.4422 & 0.1761 \\
3.0447 & -7.5922 \\
-5.5200 & 4.4900 \\
0 & 0
\end{array}\right] \\
T & =\left[\begin{array}{cc}
20 & 0 \\
0 & 15
\end{array}\right] .
\end{aligned}
$$

Notice that matrix $A$ is unstable (the eigenvalues of $A$ are: -1.9809 , $-0.3340,0.2807 \pm j 0.0952)$. Consider that the bounds on the control are given by $\rho=\left[\begin{array}{ll}5 & 2\end{array}\right]^{T}$ and $\xi=\left[\begin{array}{ll}2 & 5\end{array}\right]^{T}$ and the set of admissible initial conditions is an hypercube in $\Re^{4}: \mathcal{Z}_{0}=\left\{x \in \Re^{4}, u=0\right.$; $\left.-1 \leq x_{(i)} \leq 1, \forall i=1, \ldots, 4\right\}$. Let $\mathcal{D}_{p}$ be defined as in Example 1 .

Considering the aforementioned data, Table III shows the final values of $\underline{\alpha}, \underline{\beta}$ and $\delta$ obtained from the iterative algorithm proposed in Section $\mathrm{V}$ for different $\mu$, considering the initialization of $\underline{\alpha}$ and $\beta$ as $\left[\begin{array}{ll}1 & 1\end{array}\right]^{T} . \delta_{\text {lin }}$ corresponds to the best solution considering the linear case $\left(\underline{\alpha}=\beta=\left[\begin{array}{ll}1 & 1\end{array}\right]^{T}\right)$.

Regarding Table III, the same comments done in the previous example apply now for the multiple-input case. Note that for $\mu=0$, the best solution is obtained for the linear case. However, the poles of $(\mathcal{A}+\mathcal{B K})$ are in this case very close to the imaginary axis. On the other hand, it can be noticed that for $\mu \neq 0$, by allowing saturation, it is possible to stabilize the system for a larger set of initial conditions. 


\section{REFERENCES}

[1] H. J. Sussmann, E. D. Sontag, and Y. Yang, "A general result on the stabilization of linear systems using bounded controls," IEEE Trans. Automat. Contr., vol. 39, pp. 2411-2425, Dec. 1994.

[2] Z. Lin and A. Saberi, "Semi-global exponential stabilization of linear systems subject to input saturation via linear feedback," Syst. Control Lett., vol. 21, pp. 225-239, 1993.

[3] J. M. Gomes da Silva, Jr. and S. Tarbouriech, "Local stabilization of discrete-time linear systems with saturating controls: an LMI-based approach," IEEE Trans. Automat. Contr., vol. 46, pp. 119-125, Jan. 2001.

[4] R. B. Miller and M. Pachter, "Maneuvering flight control with actuator constraints," J. Guid., Control, Dyna., vol. 20, no. 4, pp. 22-23, July-Aug. 1997.

[5] L. Rundqwist and R. Hillgren, "Phase compensation of rate limiters in JAS-93 Grippen," in Proc. AIAA-Flight Mechanics Conf., San Diego, CA, 1996, pp. 2634-2642.

[6] Z. Lin, "Semi-global stabilization of linear systems with position and rate-limited actuators," Syst. Control Lett., vol. 30, pp. 1-11, 1997.

[7] A. A. Stoorvogel and A. Saberi, "Output regulation for linear systems to amplitude plus rate saturating actuators," Int. J. Robust Nonlinear Control, vol. 9, no. 10, pp. 631-657, 1999.

[8] G. Shi, A. Saberi, and A. A. Stoorvogel, "On $\boldsymbol{L}_{p}$ performance with global internal stability for linear systems with actuators subject to amplitude and rate saturations," in Proc. 19th Amer. Control Conf., Chicago, IL, 2000, pp. 730-734

[9] F. Tyan and D. S. Bernstein, "Dynamic output feedback compensation for linear systems with independent amplitude and rate saturation," Int. J. Control, vol. 67, no. 1, pp. 89-116, 1997.

[10] J. M. Shewchun and E. Feron, "High performance bounded control," in Proc. 16th Amer. Control Conf., vol. 5, Albuquerque, NM, June 1997, pp. 3250-3254.

[11] T. Nguyen and F. Jabbari, "Output feedback controllers for disturbance attenuation with actuator amplitude and rate saturation," Automatica, vol. 36, pp. 1339-1346, 2000.

[12] S. Tarbouriech, G. Garcia, and D. Henrion, "Local stabilization of linear systems with position and rate bounded actuators," in Proc. IFAC World Congr., Beijing, China, 1999, pp. 459-464.

[13] T. Kiyama and T. Iwasaki, "On the use of multi-loop circle for saturating control synthesis," Syst. Control Lett., vol. 41, pp. 105-114, 2000.

[14] M. Chilali and P. Gahinet, " $\boldsymbol{H}_{\infty}$ design with pole placement constraints: an LMI approach," IEEE Trans. Automat. Contr., vol. 41, pp. 358-367, Mar. 1996.

[15] J. M. Gomes da Silva, Jr., A. Fischman, S. Tarbouriech, J. M. Dion, and L. Dugard, "Synthesis state feedback for linear systems subject to control saturation by an LMI-based approach," in Proc. 2nd IFAC Symp. Robust Control, Budapest, Hungary, 1997, pp. 229-234.

[16] S. Boyd, L. El Ghaoui, E. Feron, and V. Balakrishnan, Linear Matrix Inequalities in System and Control Theory. Philadelphia, PA: SIAM, 1994, SIAM Studies in Applied Mathematics.

[17] LMI Control Toolbox User's Guide, The Math Works Inc., Natick, MA, 1995.

[18] A. Bateman and Z. Lin, "An analysis and design method for linear systems under nested saturation," in Proc. Amer. Control Conf., Ankorage, AK, 2002, pp. 1198-1203.

[19] J. M. Gomes da Silva, Jr. and S. Tarbouriech, "Local stabilization of linear systems under amplitude and rate saturating actuators," in Proc. 39th IEEE Conf. Decision Control, Sydney, Australia, 2000, pp. $3260-3265$.

\section{Solving the Scalar Feedback Nash Algebraic Riccati Equations: An Eigenvector Approach}

\author{
Jacob C. Engwerda
}

\begin{abstract}
In this note, we present an algorithm to compute all solutions of the scalar algebraic Riccati equations that play an important role in finding feedback Nash equilibria of the scalar $N$-player linear-quadratic differential game. We show that all appropriate solutions can be obtained by analyzing the eigenstructure of a related matrix.
\end{abstract}

Index Terms-Eigenvalue problem, feedback Nash equilibria, nonzero-sum linear quadratic differential games, Riccati equations.

\section{INTRODUCTION}

A well-known open problem in the area of differential games is to find a numerical algorithm to calculate all stabilizing solutions of the feedback Nash algebraic Riccati equations (AREs) (see, e.g., [2, Sec. 6.5] for precise definitions and survey of relevant literature). Each stabilizing solution of these equations determines a so-called feedback Nash equilibrium in a noncooperative linear quadratic differential game. Problems of this type were first analyzed by Case [4] and Starr and Ho [15]. Nowadays, these games are, e.g., often used in studying problems in the area of environmental economics and macroeconomic policy coordination (see, e.g., [6] for references). For the multivariable case a number of algorithms have been proposed for calculating a solution of the ARE (see, e.g., [8], [16], [13], and [9]). All these algorithms have in common that whenever they converge, they only provide one solution of the ARE. Obviously, particularly when there is no additional information that a certain type of equilibrium point is preferred, one would like to have an overview of all possible equilibria. In this note, we will present an eigenvalue-based algorithm which gives us all equilibrium points. In [1] (see also [5]), it was shown that the solutions of the ARE corresponding with the open-loop Nash game can be obtained by analyzing the eigenstructure of a matrix that can be deduced from the system matrices. Papavassilopoulos et al. considered in [14] already a similar geometric approach for the feedback Nash ARE. Their approach requires the finding of subspaces which satisfy simultaneously some invariance properties. However, up to now, it is unknown how to find these subspaces. Two other different interesting methods that have been proposed in the past for finding all isolated solutions to a system of polynomial constraints over real numbers are interval methods (see, e.g., [17] for references) and continuation methods (see, e.g., [12] and [18]). Continuation methods have been shown to be effective for problems for which the total degree is not too high, since the number of paths explored depends on the estimation of the number of solutions. Interval methods are generally robust but used to be slow. The recent approach taken in [17], however, seems to overcome this bottleneck and be rather efficient. ${ }^{1}$

In this note, we present an algorithm which is comparable to the aforementioned eigenstructure algorithm for determining the solutions of the open-loop ARE. Again, first one has to construct from the system parameters a certain matrix $M$. The equilibria of the game can then

Manuscript received June 29, 2001; revised September 6, 2002. Recommended by Associate Editor C. D. Charalambous.

The author is with the Department of Econometrics and O.R., Tilburg University, 5000 LE Tilburg, The Netherlands (e-mail: engwerda@uvt.nl).

Digital Object Identifier 10.1109/TAC.2003.811266

${ }^{1}$ I would like to thank one of the referees for pointing this out to me. 